PHYSICAL REVIEW D 95, 099903(E) (2017)

\title{
Erratum: Search for heavy neutrinos at Belle [Phys. Rev. D 87, 071102(R) (2013)]
}

\author{
D. Liventsev et al. \\ (Belle Collaboration)
}

(Received 9 May 2017; published 31 May 2017)

DOI: 10.1103/PhysRevD.95.099903

The number of neutrinos detected in the Belle detector is [Eq. (2) of the original paper]:

$$
\begin{aligned}
n\left(\nu_{h}\right) & =2 N_{B B} \mathcal{B}\left(B \rightarrow \nu_{h}\right) \mathcal{B}\left(\nu_{h} \rightarrow \ell \pi\right) \int \frac{m \Gamma}{p} \exp \left(-\frac{m \Gamma R}{p}\right) \varepsilon(R) d R \\
& =\left|U_{\alpha}\right|^{2}\left|U_{\beta}\right|^{2} 2 N_{B B} f_{1}(m) f_{2}(m) \frac{m}{p} \int \exp \left(-\frac{m \Gamma R}{p}\right) \varepsilon(R) d R
\end{aligned}
$$

where $N_{B B}$ is the number of $B \bar{B}$ pairs, $\mathcal{B}\left(B \rightarrow \nu_{h}\right)$ is the total branching fraction for $\nu_{h}$ production, $\mathcal{B}\left(\nu_{h} \rightarrow \ell \pi\right)$ is the branching fraction of the reconstructed decay, $\varepsilon(R)$ is the reconstruction efficiency of the $\nu_{h}$ decaying at a distance $R$ from the interaction point, $m, p$ and $\Gamma$ are the mass, momentum and full width of the heavy neutrino, respectively. Integration is performed over the full volume used to reconstruct the heavy neutrino vertex, which depends on reconstruction requirements. Additionally, to factor out the $|U|^{2}$ dependence, we define $\left|U_{\alpha}\right|^{2} f_{1}(m) \equiv \mathcal{B}\left(B \rightarrow \nu_{h}\right)$ and $\left|U_{\beta}\right|^{2} f_{2}(m) \equiv \Gamma\left(\nu_{h} \rightarrow \ell \pi\right)=\mathcal{B}\left(\nu_{h} \rightarrow \ell \pi\right) \Gamma$, where $\alpha$ and $\beta$ denote the flavor of the charged lepton produced in the $B$ and $\nu_{h}$ decays, respectively.

In the original paper, the exponent in the integrand was approximated by unity under the assumption that $\Gamma$ is small and the flight length is sufficiently long:

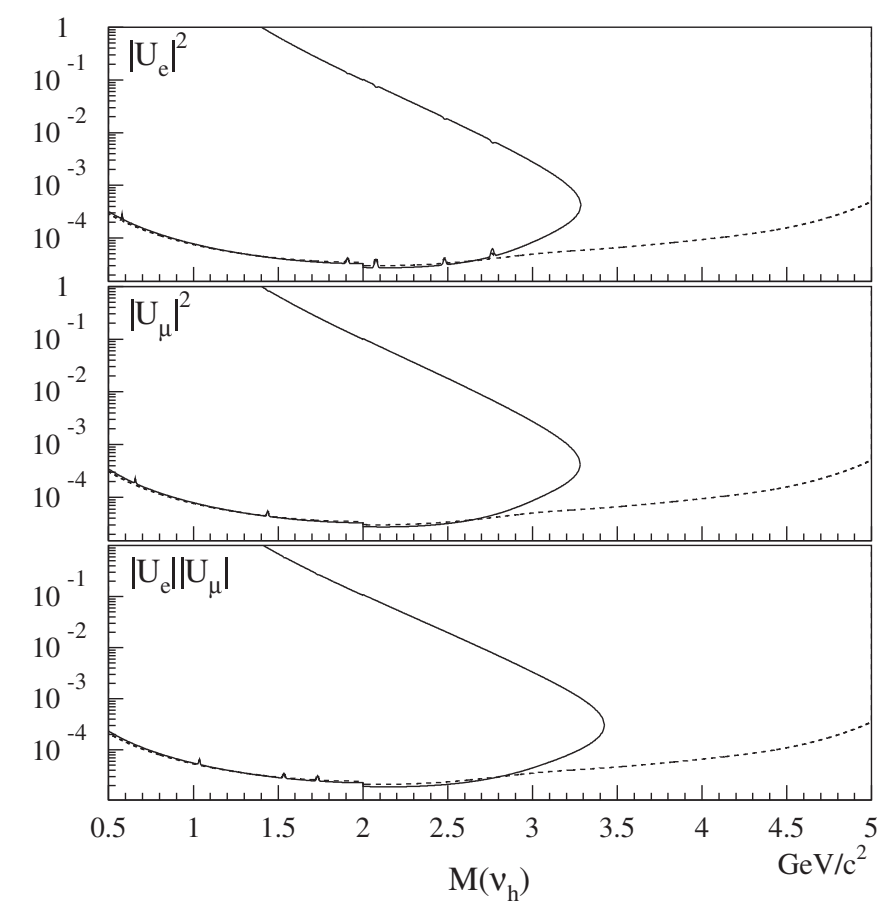

FIG. 1. Upper limits at $90 \% \mathrm{CL}$ on the $\left|U_{e}\right|^{2},\left|U_{\mu}\right|^{2}$, and $\left|U_{e}\right|\left|U_{\mu}\right|$ The solid line shows the correct exclusion region; the dashed line shows the one from the original paper. 


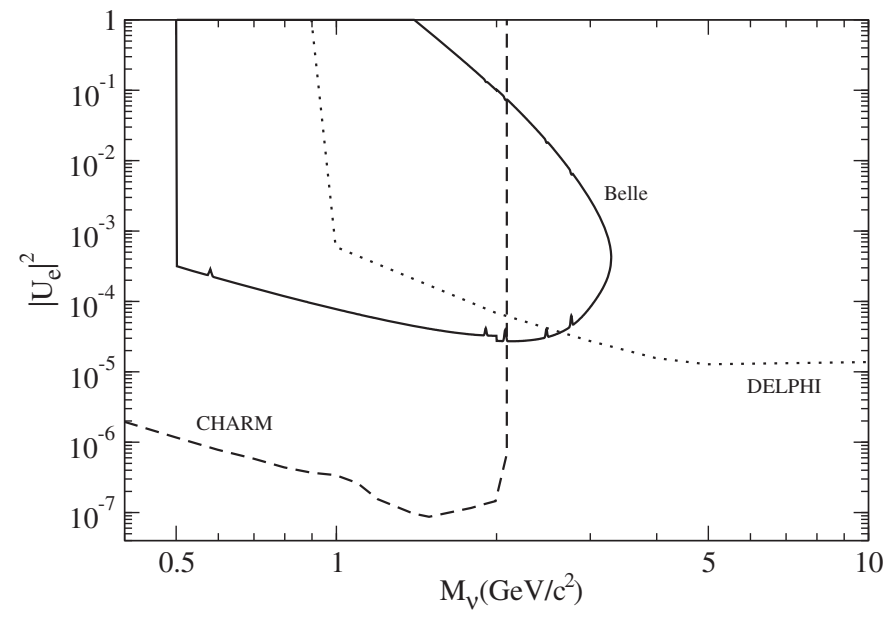

(a)

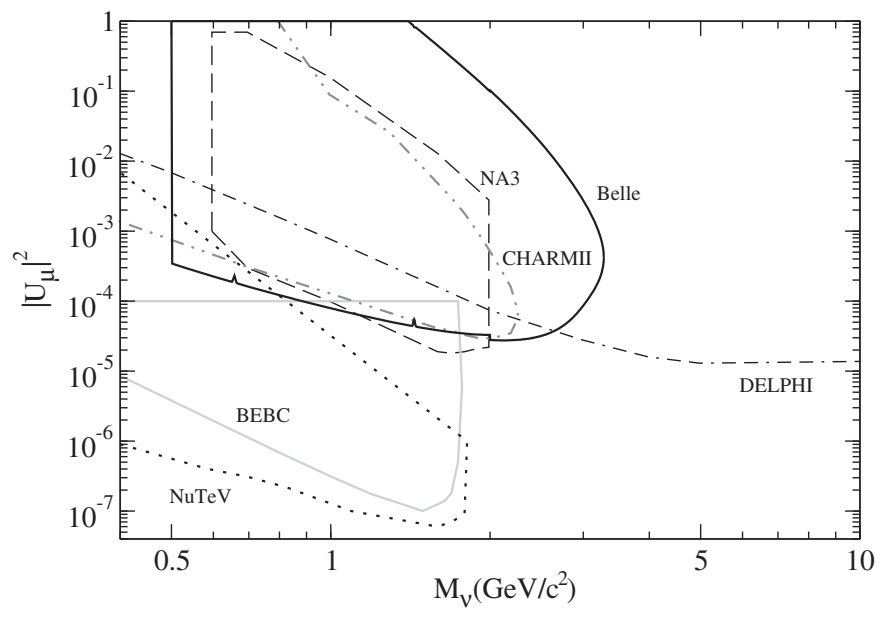

(b)

FIG. 2. Comparison of the obtained upper limits for $\left|U_{e}\right|^{2}$ (a) and $\left|U_{\mu}\right|^{2}$ (b) with existing experimental results from CHARM, CHARMII, DELPHI, NuTeV, BEBC, and NA3.

$$
n\left(\nu_{h}\right) \simeq\left|U_{\alpha}\right|^{2}\left|U_{\beta}\right|^{2} 2 N_{B B} f_{1}(m) f_{2}(m) \frac{m}{p} \int \varepsilon(R) d R
$$

Here, the momentum of the heavy neutrino was kept constant.

The assumption of a long flight length is valid only for a small neutrino mass. However, since the partial decay widths approximately increase with mass as $m^{3}$ to $m^{5}$ depending on the final state [1], at higher masses this approximation introduces a significant overestimation of the upper limit on $|U|^{2}$. Moreover, at large masses there is also an upper bound of the excluded region since, for some values of the coupling constant, the flight length is so small that the event is rejected by the selection criteria. Therefore, we recalculate the upper limits on $|U|^{2}$ taking into account the exponent in Eq. (2) and the actual neutrino momentum. The full neutrino width $\Gamma$ is calculated as the sum of partial widths from Ref. [1]. Since we do not know the relation among the different $U_{\alpha}$ we assume $|U|^{2}=\left|U_{\alpha}\right|\left|U_{\beta}\right|$ in the calculation of $\Gamma$. To obtain the upper limit on the coupling constants, we solve the Eq. (2) for the variable $|U|^{2}$.

The updated versions of Figs. 4(b) and 5 in the original paper with the new calculation are shown here as Figs. 1 and 2 , respectively.

We thank Brian Shuve and Michael Peskin for pointing out the issue.

[1] D. Gorbunov and M. Shaposhnikov, J. High Energy Phys. 10 (2007) 015. 